\title{
The Unilateral Nigral Lesion Induces Dramatic Bilateral Modification on Rat Brain Monoamine Neurochemistry
}

\author{
Massimo Pierucci, ${ }^{a}$ Vincenzo Di Matteo, ${ }^{a}$ Arcangelo Benigno, ${ }^{b}$ \\ Giuseppe Crescimanno, ${ }^{b}$ Ennio Esposito, ${ }^{a}$ \\ and Giuseppe Di Giovanni ${ }^{b}$
}

${ }^{a}$ Istituto di Ricerche Farmacologiche Mario Negri, Consorzio Mario Negri Sud, Santa Maria Imbaro (Chieti), Italy

${ }^{b}$ Dipartimento di Medicina Sperimentale, Sezione di Fisiologia Umana, "G. Pagano," Università degli Studi di Palermo, Palermo, Italy

\begin{abstract}
6-Hydroxydopamine (6-OHDA) is a neurotoxic compound commonly used to induce dopamine (DA) depletion in the nigrostriatal system, mimicking Parkinson's disease $(P D)$ in animals. The aim of the present study was to evaluate the 7-day effect of unilateral nigral lesion on rat brain monoamine neurochemistry. Five brain regions were examined: the brain stem, cerebellum, hippocampus, striatum, and cortex. 6-OHDAunilateral lesion dramatically modified DA, serotonin (5-HT) and their metabolites contents in both sides of the different brain nuclei. Furthermore, unilateral 6-OHDA lesion reduced DA and 5-HT contents and produced a robust inversion of their turnover in the nonlesioned side compared to sham-operated rats. These data suggest that 6-OHDA unilateral nigral lesion produces bilateral monoamine level modifications, and this piece of evidence should be taken into account when one interprets data from animal models of unilateral PD.
\end{abstract}

Key words: Parkinson's disease (PD); high performance liquid chromatography (HPLG); striatum; brain stem; cerebellum; cortex; hippocampus; 6-OHDA; monoamines

\section{Introduction}

Parkinson's disease (PD) is the second most common neurodegenerative disease, characterized by the loss of $50-70 \%$ of dopaminergic (DAergic) neurons located in the substantia nigra pars compacta $(\mathrm{SNc})$, with a concomitant reduction of striatal DA levels. ${ }^{1-6}$ Current evidence suggests an involvement of both environmental and genetic factors in the progression of PD. Important factors include: formation of free radicals, impaired mitochondrial activity,

Address for correspondence: Giuseppe Di Giovanni, Ph.D., Dipartimento di Medicina Sperimentale, Sezione di Fisiologia Umana, "G. Pagano," Università degli Studi di Palermo, Corso Tuköry 129, 90134 Palermo, Italy. Voice: +39-0916555821; fax: +39-0916555823. g.digiovanni@unipa.it increased sensitivity to apoptosis, excitoxicity, and inflammation., ${ }^{3,-10}$ Nevertheless, the specific etiology of PD is still unknown. Research on the pathogenesis of PD has rapidly advanced due to the development of animal models that have been shown to be not only essential for better understanding of the pathogenesis and progression of PD but of paramount importance for testing therapeutic agents for the treatment of PD patients..$^{2,8,9,11-17}$

Several toxin-induced animal models simulate motor deficits occurring in PD. The most frequently used toxins in PD models are the neurotoxin 1-methyl-4-phenyl1,2,3,6-tetrahydropyridine (MPTP), ${ }^{13}$ or 6hydroxydopamine (6-OHDA). ${ }^{11,14,18}$ Although MPTP is a valuable model of PD especially in 
nonhuman primates, its use has some caveats. Indeed, MPTP injection causes a bilateral Parkinson syndrome and, more importantly, a spontaneous recovery of Parkinsonian symptoms might occur. ${ }^{19,20}$

On the other hand, the 6-OHDA PD-model leads to stable motor deficits over time due to a permanent degeneration of virtually all DAergic neurons in the $\mathrm{SNc}$ after intracerebral injection of 6-OHDA into the rat nigrostriatal pathway. $^{21}$

6-OHDA is a hydroxylated analogue of the natural neurotransmitter DA. ${ }^{18}$ and selectively destroys catecholaminergic neurons. ${ }^{22}$ Inside neurons, 6-OHDA accumulates in the cytosol and induces cell death and exhibits hallmarks of apoptosis. ${ }^{23}$ As far as mechanisms underlying toxicity of 6-OHDA are concerned, participation of oxidative stress leading to excessive generation of free radicals and cell death is firmly established. $^{21}$ The degeneration of dopaminergic neurons in the $\mathrm{SNc}$ and the resulting loss of their nerve terminals in the striatum are responsible for most of the motor disturbances seen in PD.

In the present study we injected the 6OHDA unilaterally into the $\mathrm{SNc}$ to achieve directly DAergic cell loss. We performed quantitative postmortem investigations of 6OHDA effect, measuring tissue levels of brain monoamines and their metabolites such as DA, serotonin (5-HT), dihydroxyphenylacetic acid (DOPAC), homovanilic acid (HVA), and 5hydroxyindoleacetic acid (5-HIAA) by means of high performance liquid chromatography (HPLC). The areas of investigation were: cerebellum, brain stem, striatum, hippocampus, and cerebral cortex. Monoamine levels were dramatically modified by 6-OHDA lesion not only in the ipsilateral side but, strikingly, in the contralateral side compared to the values of sham-operated rats. These findings are discussed in terms of the current state of knowledge of PD pathophysiology and we suggest that they should be taken into account when one interprets data from animal models of unilateral PD.

\section{Materials and Methods}

Male Sprague-Dawley rats from Charles River Laboratories (Calco, Varese, Italy) were housed at appropriate environmental conditions $\left(21 \pm 2{ }^{\circ} \mathrm{C}\right.$ room temperature, 12-h light/dark cycle, 40-60\% humidity). Water and food was provided ad libitum. Procedures involving animals and their care were conducted in accordance with the institutional guidelines that are in compliance with national (D.L. n. 116, G.U., suppl. 40, 18 Febbraio 1992) and international laws and policies (EEC Council Directive 86/609, OJ L 358,1, Dec. 12, 1987; NIH Guide for the Care and Use of Laboratory Animals, NIH Publication N. 85-23, 1985 and Guidelines for the Use of Animals in Biomedical Research, Thromb. Haemost. 58, 10781084, 1987).

Rats were anesthetized with chloral hydrate (400 mg/kg i.p.) and placed on a stereotaxic instrument (David Kopf Instruments, Tujunga, CA, USA). The 6-OHDA was dissolved in $0.9 \%$ saline solution of ascorbic acid $(0.05 \%)$. 6-OHDA ( $5 \mu \mathrm{g}$, free base) in a volume of $5 \mu \mathrm{l}$ was infused into the left $\mathrm{SNc}(\mathrm{AP}=-5.2$, $\mathrm{L}=2.0, \mathrm{~V}=-7.5$ from the dura surface and respect to the bregma). ${ }^{24}$ Control rats received only the ascorbic acid solution. A week after the 6-OHDA lesion, vehicle and 6-OHDA-treated rats were killed by decapitation. Brains were rapidly removed, striata, hippocampi, brain stems, cortices, and cerebelli were dissected, frozen on dry ice, and stored at $-80^{\circ} \mathrm{C}$ until assay. Tissue samples were homogenized in $1 \mathrm{ml} 0.1 \mathrm{~N}$ perchloric acid and $0.45 \mu \mathrm{M}$ of 3,4-dihydroxybenzylamine (DHBA) and afterwards centrifuged for $15 \mathrm{~min}$ at 12,000 rotations/min. An aliquot of the supernatant was filtered and transferred to an Eppendorf tube. Levels of 5-HT, 5-HIAA, DA, DOPAC, and HVA were measured by reversed-phase HPLC with electrochemical detection.

The mobile phase was composed of $24 \mathrm{mM}$ citric acid, $16 \mathrm{mM} \mathrm{Na} \mathrm{HPO}_{4}$, $1.22 \mathrm{mM}$ heptanesulphonic acid sodium salt, 
$0.19 \mathrm{mM}$ EDTA, and 17.5\% methanol, adjusted to $\mathrm{pH} 2.8$ with orthophosphoric acid. This mobile phase was delivered at $1 \mathrm{ml} / \mathrm{min}$ flow rate (Pump 420, Kontron Instruments, Milano, Italy) through a Supelcosyl LC-8 column (C8, $4.0 \times 250$ mm, $5 \mu \mathrm{m}$, Supelco, Bellefonte, PA, USA). Samples were injected manually into the HPLC, and detection of DA or 3,4-DHBA was carried out with a coulometric detector (Coulochem II, ESA, Bedford, MA, USA) coupled to a dual electrode analytic cell (model 5014). The potential of the first electrode was set at $-175 \mathrm{mV}$ and the second at $+175 \mathrm{mV}$. Under these conditions, the sensitivity for DA was $0.35 \mathrm{pg} / 20 \mu \mathrm{l}$ with a signal to noise ratio of $3: 1$.

To ensure maximal reproducibility, 6OHDA dissolved in $0.1 \%$ ascorbate/saline was prepared in advance, filtered through $0.22-\mu \mathrm{m}$ filters, aliquoted, and stored at $-20^{\circ} \mathrm{C}$. Immediately before use aliquots were gently defrosted, loaded into a designated microperfusion pump, and protected from light. Control rats were injected with an equal volume of the vehicle only.

The concentration of each compound, i.e., DA, 5-HT, DOPAC, HVA, and 5-HIAA was calculated by comparison with the internal standard (DMBA). Ratios of metabolite level/monoamine level (DOPAC/DA, HVA/DA, 5-HIAA/5-HT) and indices of monoamine turnover were also calculated. Data correspond to Mean \pm SEM values of absolute levels obtained in each experimental group. 3,4-DHBA content in each sample was expressed as a percentage of the average baseline level calculated from three fractions collected before neurotoxin infusion. Data are expressed as Mean \pm SEM of the contents obtained in each experimental group. Data were analyzed by one-way analysis of variance (ANOVA) with repeated measures, followed by the Fisher's protected least significance difference post hoc test (Fisher's PLSD) to allow multiple comparisons between groups. All statistical analyses were performed with StatView version 5.0.1 (SAS Institute Inc., Cary, NC, USA).
TABLE 1. Concentrations ${ }^{\ddagger}$ in Various Rat Brain Regions after a Week from 6-OHDA-Induced NigralLesion

\begin{tabular}{lccc}
\hline & & 6 -OHDA & 6-OHDA \\
& Control & SX & DX \\
\hline$D A$ & & & \\
Brain stem & $503 \pm 32$ & $114 \pm 27^{* *}$ & $223 \pm 18^{\#}$ \\
Cerebellum & n.d. & n.d. & n.d. \\
Striatum & $5057 \pm 573$ & $381 \pm 88^{* *}$ & $3550 \pm 482^{\#}$ \\
Hippocampus & n.d. & n.d. & n.d. \\
Cortex & $670 \pm 25$ & $23 \pm 6^{* *}$ & $110 \pm 37^{\# \#}$ \\
5-HT & & & \\
Brain stem & $1917 \pm 213$ & $786 \pm 106$ & $636 \pm 63^{\# \#}$ \\
Cerebellum & $126 \pm 19$ & $278 \pm 19^{*}$ & $95 \pm 26^{\#}$ \\
Striatum & $668 \pm 132$ & $149 \pm 8$ & $139 \pm 39^{\# \#}$ \\
Hippocampus & $432 \pm 78$ & $98 \pm 16$ & $80 \pm 17^{\# \#}$ \\
Cortex & $924 \pm 83$ & $187 \pm 41^{*}$ & $394 \pm 184^{\# \#}$ \\
\hline
\end{tabular}

†Of dopamine (DA) and serotonin (5-HT) (ng/g of wet weight of tissue)

Data are Means \pm SEM from 10 experiments. For statistical analyses, one-way ANOVA and the Fisher's PLSD post hoc test were used. *vs. contralateral side (6-OHDA DX), ${ }^{*}$, vs. controls (sham operated); *or ${ }^{\#} P<0.05$, ${ }^{* *}$ or ${ }^{\# \#} P<0.01$.

6-hydroxydopamine hydrobromide (6OHDA) was from Aldrich Chem. Co. Milwaukee, WI, USA.

\section{Results}

Unilateral lesion dramatically modified DA and 5-HT and their metabolites levels in both sides of rat brains (Table 1). DA levels were reduced by $89.2 \pm 9.5 \%(P<0.01)$, $79.3 \pm 11.2 \%(P<0.01), 48.8 \pm 6.5 \%$ $(P<0.01)$ in the left striatum, cortex, and brain stem, respectively, compared to the right hemisphere (Table 1). Furthermore, DA levels of the right side of the lesioned rats were also reduced by $29.8 \pm 6.9 \%(P<0.01), 83.6 \pm 12.3 \%$ $(P<0.01), 55.7 \pm 9.8 \%(P<0.01)$ in the striatum (Fig. 1), cortex, and brain stem, respectively, compared to control (sham-operated) rats (Table 1). Although the 5-HT levels in the lesioned rats were similar in both sides, a very significant reduction in contents and 
Striatum

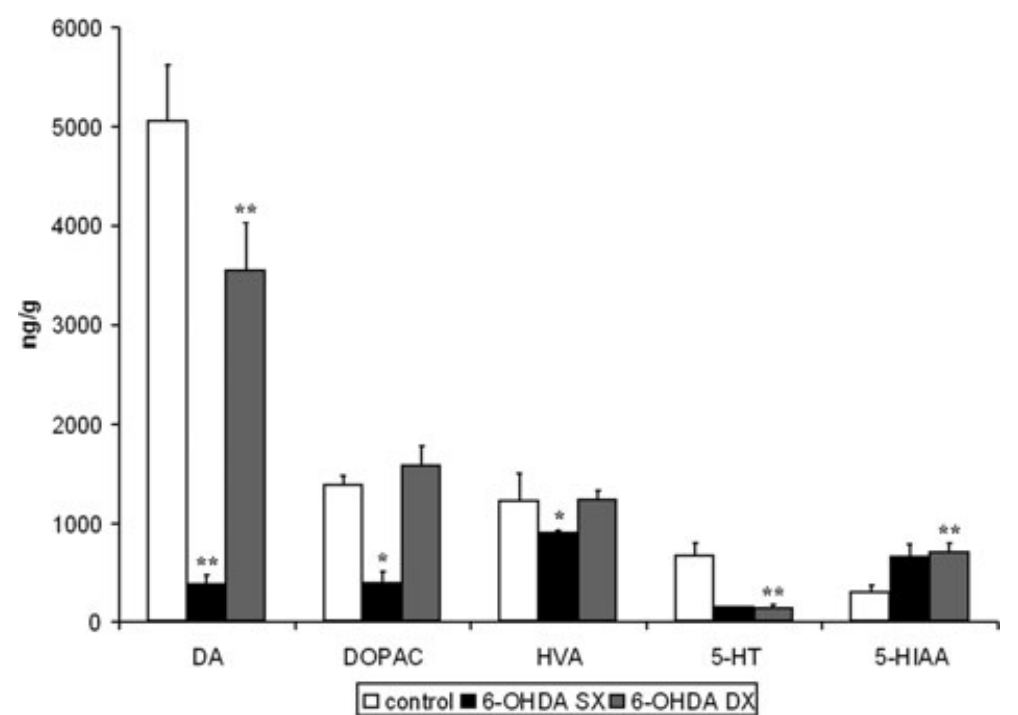

Figure 1. Effect of 6-OHDA on concentration of dopamine (DA), 3,4dihydroxyphenylacetic acid (DOPAC), homovanilic acid (HVA), serotonin (5-HT), and 5-hydroxyindoleacetic acid (5-HIAA) ( $\mathrm{ng} / \mathrm{g}$ of wet weight of tissue) in striatum of control animals and rats with 6-OHDA nigral lesion ( $n=5$, for each group). Data are Means \pm SEM. For statistical analyses one-way ANOVA and the Fisher's PLSD post hoc test were used. *vs. controlateral side (6-OHDA DX), "vs. controls (sham-operated); ${ }^{*} P<0.05,{ }^{* *}$ or ${ }^{\# \#} P<0.01$.

an inversion of 5-HT turnover (5-HIAA/5HT) compared to control rats was revealed (Table 1).

\section{Discussion}

In the present work we assessed the effect of 6-OHDA on brain areas that receive DAergic afferent such as the ventral mesenchephalon, the brain stem, striatum, hippocampus, and cortical areas. Strikingly, we showed that the modifications induced by unilateral 6-OHDA lesion are far from being a simple depletion of DA contents. Although the degree of depletion was usually less conspicuous in other brain areas than in the striatum, all areas were affected by the lesion.

In agreement with a large body of evidence, ${ }^{12,25,26}$ we confirmed that moderate ${ }^{27}$ unilateral 6-OHDA lesion of mesencephalic $\mathrm{SNc}$ neurons caused a massive depletion of striatal DA contents (up to about 90\%) when measured from the ipsilateral side compared with the contralateral side of the same animal. Moreover, DA contents were also reduced by $50-80 \%$ in the left cortex and brain stem. DAergic metabolites, DOPAC, and HVA levels showed similar reductions in the brain stem, striatum, and cortex, like their transmitter. In the striatum and cortex, DOPAC decline was less expressed than that of DA. Moreover, the metabolite/neurotransmitter ratio (DOPAC/DA) was increased, and even inverted in the striatum, compared to the contralateral side or sham-operated control. The increased DOPAC/DA ratio probably reflects increased DA turnover and DA receptor sensitization - a compensatory mechanism in surviving neurons. ${ }^{28}$ Since this ratio is used as an index of DA turnover, the alteration of this ratio in our partially lesioned rats likely reflects compensatory mechanisms involving altered DA release, reuptake, or degradation in striatal neurons. ${ }^{29,30}$ 
TABLE 2. Concentrations $\ddagger$ in Various Rat Brain Regions after a Week from 6-OHDA-Induced NigralLesion

\begin{tabular}{|c|c|c|c|}
\hline & Control & $\begin{array}{c}\text { 6-OHDA } \\
\text { SX }\end{array}$ & $\begin{array}{c}\text { 6-OHDA } \\
\text { DX }\end{array}$ \\
\hline \multicolumn{4}{|l|}{ DOPAC } \\
\hline Brain stem & $67 \pm 8$ & $106 \pm 19^{*}$ & $215 \pm 34^{\# \#}$ \\
\hline Cerebellum & n.d. & n.d. & n.d. \\
\hline Striatum & $1386 \pm 91$ & $390 \pm 12^{*}$ & $1589 \pm 192$ \\
\hline Hippocampus & n.d. & n.d. & n.d. \\
\hline Cortex & $136 \pm 13$ & $23 \pm 4^{*}$ & $93 \pm 26$ \\
\hline \multicolumn{4}{|l|}{ HVA } \\
\hline Brain stem & $54 \pm 14$ & $44 \pm 8$ & $38 \pm 8$ \\
\hline Cerebellum & n.d. & n.d. & n.d. \\
\hline Striatum & $1225 \pm 132$ & $907 \pm 25^{*}$ & $1233 \pm 103$ \\
\hline Hippocampus & n.d. & n.d. & n.d. \\
\hline Cortex & $96 \pm 11.1$ & $56 \pm 30$ & $65 \pm 13$ \\
\hline \multicolumn{4}{|l|}{ 5-HIAA } \\
\hline Brain stem & $622 \pm 120$ & $838 \pm 65$ & $995 \pm 49$ \\
\hline Cerebellum & $50 \pm 7$ & $376 \pm 22^{* *}$ & $161 \pm 23^{\#}$ \\
\hline Striatum & $307 \pm 63$ & $664 \pm 121$ & $705 \pm 99^{\# \#}$ \\
\hline Hippocampus & $179 \pm 40$ & $470 \pm 13$ & $529 \pm 15^{\# \#}$ \\
\hline Cortex & $229 \pm 11$ & $376 \pm 73$ & $476 \pm 13^{\# \#}$ \\
\hline \multicolumn{4}{|l|}{ DOPAC/DA } \\
\hline Brain stem & $0.13 \pm 0.02$ & $0.91 \pm 0.06$ & $0.96 \pm 0.18^{\# \#}$ \\
\hline Cerebellum & n.d. & n.d. & n.d. \\
\hline Striatum & $0.27 \pm 0.02$ & $1.02 \pm 0.13^{* *}$ & $0.45 \pm 0.04$ \\
\hline Hippocampus & n.d. & n.d. & n.d. \\
\hline Cortex & $0.20 \pm 0.05$ & $1.02 \pm 0.07^{* *}$ & $0.84 \pm 0.07^{\# \#}$ \\
\hline \multicolumn{4}{|l|}{ 5-HIAA/5-HT } \\
\hline Brain stem & $0.32 \pm 0.05$ & $1.06 \pm 0.06^{*}$ & $1.56 \pm 0.07^{\# \#}$ \\
\hline Cerebellum & $0.39 \pm 0.03$ & $1.35 \pm 0.12$ & $1.69 \pm 0.08^{\# \#}$ \\
\hline Striatum & $0.45 \pm 0.04$ & $4.44 \pm 1.47$ & $5.08 \pm 0.25^{\# \#}$ \\
\hline Hippocampus & $0.40 \pm 0.05$ & $4.76 \pm 0.82$ & $6.57 \pm 0.86^{\# \#}$ \\
\hline Cortex & $0.24 \pm 0.01$ & $2.01 \pm 0.18^{* *}$ & $1.20 \pm 0.06^{\# \#}$ \\
\hline
\end{tabular}

$\ddagger$ Of 3,4-dihydroxyphenylacetic acid (DOPAC), homovanilic acid (HVA), 5-hydroxyindoleacetic acid (5-HIAA) (ng/g of wet weight of tissue) and monoamine oxidase activity expressed as metabolite ratios (DOPAC/DA and 5-HIAA/5HT).

Data are means \pm SEM from 10 experiments. For statistical analyses, one-way ANOVA and the Fisher's PLSD post hoc test were used. ${ }^{*}$ vs. contralateral side (6-OHDA DX), ${ }^{\#}$ vs. controls (sham-operated); ${ }^{*}$ or ${ }^{\#} P<0.05,{ }^{* *}$ or ${ }^{\# \#} P<0.01$.

In addition, we evaluated the modification of 5-HT and 5-HIAA induced by 6-OHDA lesion in our PD model. In fact, it has been shown that 5-HT plays a role in regulating the basal ganglia circuitry, ${ }^{31,32}$ and abnormalities in 5-HT transmission might contribute to the neural mechanisms underlying PD. ${ }^{31,33,34}$ Moreover, 5-HT is involved in the movement abnormalities generally associated with the use of levodopa (L-DOPA). ${ }^{32,35,36}$ 5-HT tissue levels were unchanged in the brain stem, striatum, and hippocampus, increased in the cerebellum, and decreased in the cortex. Its metabolite 5HIAA levels were unchanged in all the areas apart from in the cerebellum, where these levels increased. 5-HT turnover was also modified; 5HIAA/5-HT ratio decreased in the brain stem and increased in the cortical areas. Contrasting 
results are reported in the literature about the effect of 6-OHDA lesion on 5-HT brain contents. ${ }^{37-40}$ It is likely that this variability is due to the amount of DA lesion achieved in the different studies.

Taken together, our data show that the specific pattern of changes in serotonergic activity, occurring after unilateral 6-OHDA lesion, is subtle compared to DA modifications.

Unilateral lesions of the nigrostriatal projection may also have contralateral consequences when compared to the nonlesioned animal. In fact, it has been shown that after unilateral electrolytical and chemical lesions DA content of the SNc decreases not only ipsilaterally but contralaterally as well. ${ }^{41}$ In agreement with this finding, we revealed a dramatic drop in DA contents in the right (nonlesioned) side when compared to the control group. This contralateral decrease was significant in the brain stem, striatum, and cortex. DOPAC and HVA levels were instead unchanged. As a consequence, in the contralateral side we observed a significant increase in DA turnover. The effect of 6-OHDA lesion was even more evident in regard to 5-HT and its metabolite. Indeed, 5-HT brain levels were decreased and the 5-HIAA/5-HT ratio, which is an expression of MAO A activity, was inverted in all the areas of examination. Strikingly, this ratio in the striatum and the hippocampus was greater than 5:1.

Therefore, the loss of DA innervation triggered an increase in DA and 5-HT turnover in all the brain. Regarding the neurochemistry of 5-HT, the increase in its turnover and the concomitant depletion might be the pathological substrate that predisposes PD patients to depression. Indeed, besides being a movement disorder, PD is also associated with numerous nonmotor symptoms. Mood disturbance, and especially major depressive disorder, has an average prevalence of $25-40 \%$ in outpatient settings. ${ }^{42}$

In summary, the present study provides, for the first time, compelling evidence that unilateral intranigral 6-OHDA infusion alters the monoamine neurochemistry of all the brain. Therefore, although the 6-OHDA model does not mimic all pathological and clinical features of human Parkinsonism, it might reproduce the complex alterations in neurotransmitter chemistry that characterize this neurological disorder. Our findings therefore suggest that 6-OHDA unilateral nigral lesion produces bilateral monoamine level modifications and this piece of evidence should be taken into account when interpreting data from animal models of unilateral PD to avoid errors in the estimation of lesion effects and, above all, the activity of neuroprotective substances.

\section{Acknowledgments}

This study was supported in part by Ateneo di Palermo research funding, project ORPA068JJ5, coordinator G. Di Giovanni.

\section{Conflicts of Interest}

The authors declare no conflicts of interest.

\section{References}

1. Hornykiewicz, O. 1963. The tropical localization and content of noradrenalin and dopamine (3hydroxytyramine) in the substantia nigra of normal persons and patients with Parkinson's disease. Wien Klin Wochenschr. 75: 309-312.

2. Esposito, E., V. Di Matteo \& G. Di Giovanni. 2007. Death in the substantia nigra: A motor tragedy. Expert Rev. Neurother. 7: 7677-7697.

3. Di Giovanni, G. 2008. Will it be possible to prevent nigral neurodegeneration? CNS Neurol. Disord. Drug Targets 7: 28-44.

4. Di Giovanni, G. 2007. The Basal Ganglia Pathophysiology: Recent Advances. Transworld Research Network. Kerala, India.

5. Jellinger, K. 1989. Pathology of Parkinson's disease. In Handbook of Experimental Pharmacology. D.B. Calne, Ed.: Vol. 8: 47-112. Springer. Berlin.

6. Scherman, D. et al. 1989. Striatal dopamine deficiency in Parkinson's disease: Role of aging. Ann. Neurol. 26: 551-557. 
7. Di Giovanni, G. et al. 2007. Biochemical and therapeutic effects of antioxidants in the treatment of Parkinson's disease. In The Basal Ganglia Pathophysiology: Recent Advances. G. Di Giovanni, Ed: 253-283 Transworld Research Network. Kerala, India.

8. Di Matteo, V. et al. 2006. 7-nitroindazole protects striatal dopaminergic neurons against MPP+-induced degeneration: An in vivo microdialysis study. Ann. $\mathcal{N}$. r. Acad. Sci. 1089: 462-471.

9. Di Matteo, V. et al. 2006. Aspirin protects striatal dopaminergic neurons from neurotoxin-induced degeneration: An in vivo microdialysis study. Brain Res. 1095: $167-177$.

10. von Bohlen und Halbach, O., A. Schober \& K. Krieglstein. 2004. Genes, proteins, and neurotoxins involved in Parkinson's disease. Prog. Neurobiol. 73: 151-177.

11. Betarbet, R., T.B. Sherer \& J.T. Greenamyre. 2002. Animal models of Parkinson's disease. Bioessays 24: 308-318.

12. Beal, M.F. 2001. Experimental models of Parkinson's disease. Nat. Rev. 2: 325-332.

13. Schober, A. 2004. Classic toxin-induced animal models of Parkinson's disease: 6-OHDA and MPTP. Cell Tissue Res. 318: 215-224.

14. Dauer, W. \& S. Przedborski. 2003. Parkinson's disease: Mechanisms and models. Neuron 39: 889 909.

15. Deumens, R., A. Blokland \& J. Prickaerts. 2002. Modeling Parkinson's disease in rats: An evaluation of 6-OHDA lesions of the nigrostriatal pathway. Exp. Neurol. 175: 303-317.

16. Glinka, Y., M. Gassen \& M. B. H. Youdim. 1997. Mechanism of 6-hydroxydopamine neurotoxicity. $\mathcal{F}$. Neural Transm. 50: 55-66.

17. Hornykiewicz, O. 1998. Biochemical aspects of Parkinson's disease. Neurology 51: S2-9.

18. Blum, D. et al. 2001. Molecular pathways involved in the neurotoxicity of 6-OHDA, dopamine and MPTP: Contribution to the apoptotic theory in Parkinson's disease. Prog. Neurobiol. 65: 135-172.

19. Eidelberg, E. et al. 1986. Variability and functional recovery in the N-methyl-4-phenyl-1,2,3,6tetrahydropyridine model of Parkinsonism in monkeys. Neuroscience 18: 817-822.

20. Taylor, J.R. et al. 1997. Severe long-term 1-methyl4-phenyl-1,2,3,6-tetrahydropyridineinduced Parkinsonism in the Vervet monkey (Cercopithecus aethiops sabaeus). Neuroscience 81: 745-755.

21. Sachs, C. \& G. Jonsson. 1975. Mechanisms of action of 6-hydroxydopamine. Biochem. Pharmacol. 24: $1-8$.

22. Luthman, J. et al. 1989. Selective lesion of central dopamine or noradrenaline neuron systems in the neonatal rat: Motor behavior and monoamine al- terations at adult stage. Behav. Brain Res. 33: 267227.

23. Liang, Q. et al. 2004. 6-hydroxydopamine induces dopaminergic cell degeneration via a caspase-9mediated apoptotic pathway that is attenuated by caspase-9dn expression. F. Neurosci. Res. 77: 747-761.

24. Paxinos, G. \& C. Watson. 1986. The Rat Brain in Stereotaxic Coordinates. Second Edition. Academic Press. New York, NY.

25. Porter, C.C., J.A. Totaro \& C.A. Stone. 1963. Effect of 6-hydroxydopamine and some other compounds on the concentration of norepinephrine in the hearts of mice. 7. Pharmacol. Exp. Ther. 140: 308-316.

26. Ungerstedt, U. 1976. 6-hydroxydopamine-induced degeneration of the nigrostriatal dopamine pathway: The turning syndrome. Pharmacol. Ther. 2: 37-40.

27. Schwarting, R.K.W. \& J.P. Huston. 1996. The unilateral 6-hydroxydopamine lesion model in behavioral brain research. Analysis of functional deficits, recovery and treatments. Prog. Neurobiol. 50: 275-331.

28. Enz, A., F. Hefti \& W. Frick. 1984. Acute administration of l-methyl-4-phenyl-1,2,3,6-tetrahydropyridine (MPTP) reduces dopamine and serotonin but accelerates norepinephrine metabolism in the rat brain. Effect of chronic pretreatment with MPTP. Eur. 7 . Pharmacol. 101: 37-44.

29. Fornaguera, J. et al. 1994. Behavioural asymmetries and recovery in rats with different degrees of unilateral striatal dopamine depletion. Brain Res. 664: 178-188.

30. Sherman, T.G. \& C.A. Moody. 1995. Alterations in tyrosine hydroxylase expression following partial lesions of the nigrostriatal bundle. Mol. Brain Res. 29: 285-296.

31. Di Giovanni, G. et al. 2001. mChlorophenylpiperazine excites non-dopaminergic neurons in the rat substantia nigra and ventral tegmental area by activating serotonin-2C receptors. Neuroscience 103: 111-116.

32. Di Matteo, V. et al. 2008. Serotonin modulation of the basal ganglia circuitry: Therapeutic implication for Parkinson's disease and other motor disorders. In Serotonin-Dopamine Interaction: Experimental Evidence and Therapeutic Relevance. Prog. Brain Res., Vol. 172. G. Di Giovanni, V. Di Matteo \& E. Esposito, Eds.: 423-463. Elsevier. Amsterdam, The Netherlands.

33. Di Giovanni, G. et al. 2006. Serotonin involvement in the basal ganglia pathophysiology: Could the 5HT2C receptor be a new target for therapeutic strategies? Curr. Med. Chem. 13: 3069-3081.

34. Hornykiewicz, O. 1989. Ageing and neurotoxins as causative factors in idiopathic Parkinson's diseaseA critical analysis of the neurochemical evidence. Prog. Neuropsychopharmacol. Biol. Psychiatry 13: 319 328. 
35. Bezard, E., J.M. Brotchie \& C.E. Gross. 2001. Pathophysiology of levodopa-induced dyskinesia: Potential for new therapies. Nat. Rev. Neurosci. 2: 577588.

36. Carta, M. et al. 2007. Dopamine released from 5-HT terminals is the cause of L-DOPA-induced dyskinesia in Parkinsonian rats. Brain 130: 1819-1833.

37. Luthman, J., M. Herrera-Marschitz \& E. Lindqvist. 1994. Unilateral neonatal intracerebroventricular 6hydroxydopamine administration in rats: I. Effects on spontaneous and drug-induced rotational behaviour and on post-mortem monoamine levels. Psychopharmacology 116: 443-50.

38. Molina-Holgado, E. et al. 1993. Changes of amino acid and monoamine levels after neonatal 6hydroxydopamine denervation in rat basal ganglia, substantia nigra and raphe nuclei. F. Neurosci. Res. 35: 409-418.
39. Breese, G.R. et al. 1984. Behavioral differences between neonatal and adult 6-hydroxydopaminetreated rats to dopamine agonists: relevance to neurological symptoms in clinical syndromes with reduced brain dopamine. 7. Pharmacol. Exp. Ther. 231: 343354.

40. Zhou, F.C., S. Bledsoe \& J. Murphy. 1991. Serotonergic sprouting is induced by dopamine-lesion in substantia nigra of adult rat brain. Brain Res. 556: 108116.

41. Hahn, Z., Z. Karadi \& L. Lenard. 1981. Striatal dopamine levels after unilateral lesions of the substantia nigra: Evidence for a contralateral decrease. Acta Physiol. Acad. Sci. Hung. 57: 249-253.

42. Miller, K.M. et al. 2007. Depression symptoms in movement disorders: Comparing Parkinson's disease, dystonia, and essential tremor. Mov. Disord. 22: 666672 . 